\title{
QUALITATIVE THEORY OF PARTIAL DIFFERENCE EQUATIONS (IV): FORCED OSCILLATIONS OF HYPERBOLIC TYPE NONLINEAR PARTIAL DIFFERENCE EQUATIONS
}

\author{
SUI SUN CHENG, BING GEN ZHANG AND SHENG-LI XIE
}

\begin{abstract}
Nonli1:ear hyperbolic type partial difference equations with a forcing term are studied in this paper. By means of two averaging techniques, the problems of oscillation of characteristic initial value problem and of initial boundary value problem are reduced to that of forced and/or unforced recurrence relations in one variable. A variety of oscillation criteria is given for these relations which in turn yield oscillation criteria for the partial difference equations.
\end{abstract}

\section{Introduction}

We are concerned with the oscillatory behavior of hyperbolic type nonlinear partial difference equations of the form

$$
\Delta_{2}^{2} u(i, j-1)-\Delta_{1}^{2} u(i-1, j)+q(i, j, u(i, j))=f(i, j), \quad 1 \leq i \leq n, j \geq 1
$$

and

$$
\Delta_{21}^{2} u(i, j)+c(i, j, u(i, j))=f(i, j), \quad i, j=0,1,2, \ldots
$$

where the partial differences $\Delta_{1} u(i, j), \Delta_{1}^{2} u(i, j), \Delta_{2} u(i, j), \Delta_{2}^{2} u(i, j), \Delta_{21}^{2} u(i, j)$ are defined respectively by

$$
\begin{array}{ll}
\triangle_{1} u(i, j)=u(i+1, j)-u(i, j), & \Delta_{1}^{2} u(i, j)=u(i+2, j)-2 u(i+1, j)+u(i, j), \\
\triangle_{2} u(i, j)=u(i, j+1)-u(i, j), & \Delta_{2}^{2} u(i, j)=u(i, j+2)-2 u(i, j+1)+u(i, j),
\end{array}
$$

and

$$
\Delta_{21}^{2} u(i, j)=u(i+1, j+1)-u(i+1, j)-u(i, j+1)+u(i, j)
$$

Received May 3, 1994; revised October 15, 1994.

1991 Mathematics Subject Classification. 39A10, 35R10.

Key words and phrases. Partial difference equations, oscillation criteria, characteristic initial value problem, initial boundary value problem. 
These two equations can respectively be considered as discrete analogs of hyperbolic differential equations of the form

$$
u_{t t}(x, t)-u_{x x}(x, t)+q(x, t, u)=f(x, t)
$$

and

$$
u_{x t}(x, t)+c(x, t, u)=f(x, t)
$$

which have been investigated in a number of recent studies $[6,11,12]$. We refer the reader to [6] for initial boundary value problems involving equations of the form $(1,3)$, and to $[6,7,12]$ for characteristic initial value problems involving equations of the form (1.4).

This work is motivated by several of our earlier works $[1,2,3]$ and by the results obtained in these studies and the fact that several averaging techniques in obtaining these results can be adopted to establish oscillation criteria for the discrete equations. Our results, however, are new and their derivations require some subtly different arguments.

Several preparatory results will be needed in the sequel.

Lemma 1.1. An eventually concave sequence $\{x(n)\}\left(\right.$ i.e. $\Delta^{2} x(n) \leq 0$ for all large $n$ ) is of constant sign eventually. An eventually positive and concave sequence (or strictly concave) is eventually nondecreasing (respectively increasing).

Lemma 1.2. ( $\mathrm{Li}$ and Cheng [9]) Let $f$ be a positive nondecreasing function defined on $(0, \infty)$, and $\{x(n)\}$ be a real sequence such that $x(k)>0$ for $i \leq k \leq j+1$, then

$$
\sum_{k=i}^{j} \frac{\Delta x(k)}{f(x(k))} \geq \int_{x(i)}^{x(j+1)} \frac{d s}{f(s)} \geq \sum_{k=i}^{j} \frac{\Delta x(k)}{f(x(k+1))} .
$$

Lemma 1.3. Let $\{q(n)\}_{0}^{\infty}$ be a sequence of nonnegative numbers with infinitely many nonzero terms and $\Sigma^{\infty} q(j)<\infty$. Then

$$
\sum_{j=0}^{\infty} q(j)\left\{\sum_{i=j+1}^{\infty} q(i)\right\}^{-1}=\infty .
$$

Proof. Let

$$
\rho(j)=\sum_{i=j+1}^{\infty} q(i), \quad j \geq 0 .
$$

Then $\{\rho(j)\}$ is a positive sequence and $\Delta \rho(j)=-q(j+1) \leq 0$ for $j \geq 0$. Thus by Lemma 1.2 ,

$$
\begin{aligned}
\sum_{j=0}^{\infty} q(j)\left\{\sum_{i=j+1}^{\infty} q(i)\right\}^{-1} & \geq-\sum_{j=1}^{n} \frac{\Delta \rho(j-1)}{\rho(j)} \\
& \geq-\int_{\rho(1)}^{\rho(n)} \frac{d s}{s}=-\ln \rho(n)+\ln \rho(1) \rightarrow \infty
\end{aligned}
$$


as $n \rightarrow \infty$.

The proof of the above Lemma can also be found in Zhang and Cheng [13, Lemma 3.1] but is included here for the sake of completeness.

\section{Initial Boundary Value Problems}

In this section, we first consider the following partial difference equation

$$
\Delta_{2}^{2} u(i, j-1)-\Delta_{1}^{2} u(i-1, j)+q(i, j, u(i, j))=f(i, j), \quad 1 \leq i \leq n, j \geq 1
$$

subject to the conditions

$$
\begin{aligned}
& \Delta_{1} u(0, j)=g(j), \quad j \geq 0 \\
& \Delta_{1} u(n, j)=h(j), \quad j \geq 0,
\end{aligned}
$$

where $f(i, j), g(j)$ and $h(j)$ are real functions defined for $1 \leq i \leq n$ and/or $j \geq 0$, and $q(i, j, u)$ is a real function defined on $\{1,2, \ldots, n\} \times\{0,1,2, \ldots\} \times R$. We shall also assume some or all of the following conditions on $q$ :

(H1) $q(i, j, u) \geq p(j) \varphi(u)$ for $1 \leq i \leq n, j \geq 0$ and $-\infty<u<\infty$, where $p(j)$ is nonnegative for $j \geq 0$ and $\varphi$ is nonnegative and convex on $(0, \infty)$;

(H2) $q(i, j,-u)=-q(i, j, u)$ for $1 \leq i \leq n, j \geq 0$ and $-\infty<u<\infty$;

(H3) $\varphi$ is nondecreasing on $(0, \infty)$;

(H4) $\varphi$ is positive on $(0, \infty)$.

A solution $u(i, j)$ of (2.1-2.3) is a double sequence defined for $0 \leq i \leq n+1$ and $j \geq 0$ which satisfies (2.1), (2.2) and (2.3) respectively. Given an arbitrary function $\psi(i, j)$ defined for $0 \leq j \leq 1$ and $1 \leq i \leq n$, we can easily show by induction that a unique solution of $(2.1-2.3)$ exists which satisfies $u(i, j)=\psi(i, j)$ for $0 \leq j \leq 1$ and $1 \leq i \leq n$.

Let $w(i, j)$ be a double sequence defined for $0 \leq i \leq n+1$ and $j \geq 0$. Suppose there is some nonnegative integer $T$ such that $w(i, j)>0$ for $1 \leq i \leq n$ and $j \geq T$, then $w(i, j)$ is said to be eventually positive. An eventually negative $w(i, j)$ is similarly defined. The function $w(i, j)$ is said to be oscillatory for $1 \leq i \leq n$ and $j \geq 0$ if it is neither eventually positive nor eventually negative.

Associated with every solution $\{u(i, j)\}$ of (2.1-2.3), define

$$
U(j)=\frac{1}{n} \sum_{i=1}^{n} u(i, j), \quad j \geq 0 .
$$

Theorem 2.1. Suppose $(\mathrm{H} 1)$ holds. Let $u(i, j)$ be an eventually positive solution of $(2.1-2.3)$, then the function $U(j)$ defined by $(2.4)$ satisfies the recurrence relation

$$
\Delta^{2} U(j-1)+p(j) \varphi(U(j)) \leq F(j)
$$


for all large $j$, where

$$
F(j)=\frac{1}{n}\left\{\sum_{i=1}^{n} f(i, j)+h(j)-g(j)\right\} .
$$

In addition, suppose $(\mathrm{H} 2)$ is satisfied. Then when $v(i, j)$ is an eventually negative solution of $(2.1-2.3)$, the recurrence relation

$$
\Delta^{2} W(j-1)+p(j) \varphi(W(j)) \leq-F(j), \quad j \geq 1,
$$

has an eventually positive solution.

Proof. After summing equation (2.1) with respect to the first variable from $i=1$ to $i=n$, and then dividing through by $n$, we obtain

$$
\Delta^{2} U(j-1)=\frac{h(j)-g(j)}{n}+\sum_{i=1}^{n} \frac{f(i, j)}{n}-\sum_{i=1}^{n} \frac{q(i, j, u(i, j))}{n} .
$$

By the convexity of $\varphi$, we have

$$
\sum_{i=1}^{n} \frac{q(i, j, u(i, j))}{n} \geq p(j) \sum_{i=1}^{n} \frac{\varphi(u(i, j))}{n} \geq p(j) \varphi(U(j)),
$$

thus

$$
\Delta^{2} U(j-1)+p(j) \varphi(U(j)) \leq F(j)
$$

for all large $j$.

Next, if in additionn, (H2) holds and if $v(i, j)$ is an eventually negative solution, then $-v(i, j)$ is an eventually positive solution of

$$
\begin{gathered}
\Delta_{2}^{2} w(i, j-1)-\Delta_{1}^{2} w(i-1, j)+q(i, j, w(i, j))=-f(i, j), \quad 1 \leq i \leq n, j \geq 1 \\
\Delta_{1} w(0, j)=-g(j), \quad j \geq 0,
\end{gathered}
$$

and

$$
\Delta_{1} w(n, j)=-h(j), \quad j \geq 0 .
$$

The proof now follows by applying the first part of our Theorem.

In view of the above Theorem, in order to obtain oscillation theorems for (2.1), we need to consider recurrence relations of the following form

$$
\Delta^{2} U(j-1)+p(j) \varphi(U(j)) \leq \Psi(j), \quad j \geq 1 .
$$

A simple result is as follows. 
Lemma 2.1. Assume that $p(j) \geq 0$ for $j \geq 0$ and $\varphi$ is nonnegative on $(0, \infty)$. If

$$
\liminf _{k \rightarrow \infty} \frac{1}{k-N+1} \sum_{n=N}^{k} \sum_{j=N}^{n} \Psi(j)=-\infty
$$

then the recurrence relation (2.6) cannot have an eventually positive solution.

Proof. From (2.6), we see that if $U(j)$ is an eventually positive solution, then

$$
\Delta^{2} U(j-1) \leq \Psi(j)-p(j) \varphi(U(j)) \leq \Psi(j)
$$

for all large $j$. After summing, we have

$$
\Delta U(n) \leq \Delta U(N-1)+\sum_{j=N}^{n} \Psi(j), \quad n \geq N
$$

where $N$ is some large integer. Another summation will then yield

$$
U(k+1) \leq U(N)+(k+1-N) \Delta U(N-1)+\sum_{n=N}^{k} \sum_{j=N}^{n} \Psi(j), \quad k \geq N .
$$

Dividing the above inequality by $k+1-N$ and taking limit inferior on both sides, we see that

$$
0 \leq \Delta U(N-1)+\liminf _{k \rightarrow \infty} U(k+1) /(k-N+1) \leq-\infty,
$$

which is a contradiction.

A more complicated result is as follows.

Lemma 2.2. Assume that $p(j) \geq 0$ for $j \geq 0$ and $\varphi$ is nonnegative and nondecreasing on $(0, \infty)$. Assume further that there is a sequence $\{v(j)\}$ which has a nonpositive subsequence $\left\{v\left(j_{k}\right)\right\}$ and satisfies $\Delta^{2} v(j-1)=\Psi(j)$ for all large $j$, and that

$$
\liminf _{k \rightarrow \infty} \frac{1}{k-N+1} \sum_{n=N}^{k} \sum_{j=N}^{n} \Psi(j)-p(j) \varphi(\max (v(j), 0))=-\infty .
$$

Then (2.6) cannot have an eventually positive solution.

Proof. Suppose $U(j)$ is an eventually positive of (2.6), then $z(j)=U(j)-v(j)$ satisfies

$$
\Delta^{2} z(j-1)=\Delta^{2} U(j-1)-\Delta^{2} v(j-1) \leq-p(j) \varphi(U(j)) \leq 0
$$

for all large $j$. The sequence $\{z(j)\}$ is thus concave and hence by Lemma 1.1 , it is eventually of constant sign. The sequence $\{z(j)\}$ is eventually positive, otherwise

$$
0<U\left(j_{k}\right) \leq v\left(j_{k}\right)<0
$$


which is a contradiction. This implies

$$
U(j)>\max \{v(j), 0\}
$$

for all large $j$. By repeated summation of (2.10) (as in the derivation of (2.8)), we obtain

$$
U(k+1) \leq U(N)+(k+1-N) \Delta U(N-1)+\sum_{n=N}^{k} \sum_{j=N}^{n} \Psi(j)-p(j) \varphi(U(j))
$$

By the monotonicity of $\varphi$, we then see that

$$
\begin{aligned}
\frac{U(k+1)}{k+1-N} \leq & \frac{U(N)}{k+1-N}+\Delta U(N-1) \\
& +\frac{1}{k+1-N} \sum_{n=N}^{k} \sum_{j=N}^{n} \Psi(j)-p(j) \varphi(\max (v(j), 0)) .
\end{aligned}
$$

Taking limit inferior on both sides of above inequality, we then arrive at a contradiction.

A variant of the above result is as follows.

Lemma 2.3. Assume that $p(j) \geq 0$ for $j \geq 0$ and $\varphi$ is nonnegative and nondecreasing on $(0, \infty)$. Assume further that there is a sequence $\{v(j)\}$ which has a nonpositive subsequence $\left\{v\left(j_{k}\right)\right\}$ and satisfies $\Delta^{2} v(j-1)=\Psi(j)$ for all large $j$ and

$$
\liminf _{n \rightarrow \infty} v(n)=0 .
$$

If (2.6) has an eventually positive solution, so does the recurrence relation

$$
\Delta^{2} W(j-1)+p(j) \varphi(W(j)) \leq 0, \quad j \geq 0
$$

Proof. We have already seen in the last proof that the sequence $\{z(j)\}$ defined by $z(j)=U(j)-v(j)$ is eventually positive. By Lemma 1.1, $\{z(j)\}$ is eventually nondecreasing. After summing (2.10), we obtain

$$
\Delta z(i) \geq-\Delta z(k)+\Delta z(i) \geq \sum_{j=i+1}^{k} p(j) \varphi(U(j))
$$

and thus

$$
\Delta z(i) \geq \sum_{j=i+1}^{\infty} p(j) \varphi(U(j))
$$


Another summation then yields

$$
U(k+1)-v(k+1)=z(k+1) \geq z(K)+\sum_{i=K}^{k} \sum_{j=i+1}^{\infty} p(j) \varphi(U(j))
$$

where $K$ is taken to be a large integer so that $z(K)>0$. Since (2.11) holds, we have

$$
U(k+1) \geq \frac{z(K)}{2}+\sum_{i=K}^{k} \sum_{j=i+1}^{\infty} p(j) \varphi(U(j))
$$

for all large $k$. Let

$$
W(k+1)=\sum_{i=K}^{k} \sum_{j=i+1}^{\infty} p(j) \varphi(U(j))
$$

then

$$
\begin{aligned}
& 0<W(k+1) \leq U(k+1), \\
& \Delta W(k)=\sum_{j=k+1}^{\infty} p(j) \varphi(U(j))
\end{aligned}
$$

and

$$
\Delta^{2} W(k-1)=-p(k) \varphi(U(k))
$$

for all large $k$. Since $\varphi$ is nondecreasing, thus

$$
0=\Delta^{2} W(j-1)+p(j) \varphi(U(j)) \geq \Delta^{2} W(j-1)+p(j) \varphi(W(j))
$$

for all large $j$.

Based on ideas similar to that used above, we can prove the following result.

Lemma 2.4. Assume that $p(j) \geq 0$ for $j \geq 0$ and $\varphi$ is nonnegative and nondecreasing on $(0, \infty)$. Assume further that there is a sequence $\{v(j)\}$ which has a constant subsequence $\left\{v\left(j_{k}\right)\right\} \equiv\{h\}$ and $v(j) \geq h$ for all large $j$. Assume further that $\Delta^{2} v(j-1)=\Psi(j)$ for all large $j$. If (2.6) has an eventually positive solution $\{U(j)\}$, then so does the recurrence relation (2.12).

Proof. Since $\Delta^{2}(U(j)-v(j)+h)=\Delta^{2}(U(j)-v(j)) \leq 0$ for all large $j$, by means of Lemma 1.1, $\{U(j)-v(j)+h\}$ is eventually of constant sign. We assert that $U(j)-v(j)+h>0$ for all large $j$ for otherwise $U(j)-v(j)+h \leq 0$ would imply

$$
0<U\left(j_{k}\right)=U\left(j_{k}\right)-v\left(j_{k}\right)+h \leq 0
$$

which is contradiction. If we now set

$$
W(j)=U(j)-v(j)+h, \quad j \geq 0,
$$


then by the monotonicity of $\varphi$, we have

$$
\Delta^{2} W(j-1)+p(j) \varphi(W(j)) \leq p(j)\{\varphi(U(j)-v(j)+h)-\varphi(U(j))\} \leq 0
$$

for all large $j$. This completes the proof.

\section{Characteristic Initial Value Problems}

In this section, we consider the following partial difference equation

$$
\Delta_{21}^{2} u(i, j)+c(i, j, u(i, j))=f(i, j), \quad i, j=1,2, \ldots
$$

subject to the conditions

$$
\begin{aligned}
& \Delta_{2} u(0, j)=g(j), \quad j \geq 0 \\
& \Delta_{1} u(i, 0)=h(i), \quad i \geq 0,
\end{aligned}
$$

where $f(i, j)$ is real function defined for $i, j \geq 1, g(j)$ and $h(j)$ are real functions defined for $j \geq 0$, and $c(i, j, u)$ is a real function defined on $\{1,2, \ldots\} \times\{1,2, \ldots\} \times R$. We shall also assume some or all of the following conditions on $c(i, j, u)$ :

(G1) $c(i, j, u) \geq p(i+j) \varphi(u)$ for $i, j \geq 1$ and $-\infty<u<\infty$, where $p(n)$ is nonnegative for $n \geq 0$ and $\varphi$ is nonnegative and convex on $(0, \infty)$;

(G2) $c(i, j, u)=-c(i, j,-u)$ for $i, j \geq 1$ and $-\infty<u<\infty$;

(G3) $\varphi$ is nondecreasing on $(0, \infty)$;

(G4) $\varphi$ is positive on $(0, \infty)$.

A. solution $u(i, j)$ of (3.1-3.3) is a double sequence defined for $i \geq 0$ and $j \geq 0$ which satisfies (3.1-3.3) respectively. Given an arbitrary number $u_{0}$, we can easily show by induction that a unique solution of (3.1-3.3) exists which satisfies $u(0,0)=u_{0}$.

Let $w(i, j)$ be a double sequence defined for $i \geq 0$ and $j \geq 0$. Suppose there is some nonnegative integer $T$ such that $w(i, j)>0$ for $i+j \geq T, i \geq 1$ and $j \geq 1$, then $w(i, j)$ is said to be eventually positive. An eventually negative $w(i, j)$ is defined in a similar way. The function $w(i, j)$ is said to be oscillatory for $i, j \geq 1$ if it is neither eventually positive nor eventually negative. Note that the definitions made here are different from those made in the previous section.

Associated with every solution $\{u(i, j)\}$ of $(3.1)$, we set

$$
U(n)=\frac{1}{n+1} \sum_{k=0}^{n} u(n-k, k), \quad n \geq 1 .
$$

Then it is easily verified that

$$
\Delta((n+1) U(n))=u(0, n+1)+\sum_{k=0}^{n} \Delta_{1} u(n-k, k)
$$


and

$$
\Delta^{2}((n+1) U(n))=\Delta_{2} u(0, n+1)+\Delta_{1} u(n+1,0)+\sum_{k=0}^{n} \Delta_{21}^{2} u(n-k, k) .
$$

Theorem 3.1. Suppose (G1) holds. Let $u(i, j)$ be an eventually positive solution of (3.1-3.3), then the function $U(n)$ defined by (3.4) satisfies the recurrence relation

$$
\Delta^{2}((n+1) U(n))+(n+1) p(n) \varphi(U(n)) \leq G(n)
$$

for all large $n$, where

$$
G(n)=g(n+1)+h(n+1)+\sum_{k=0}^{n} f(n-k, k), \quad n \geq 1
$$

In addition, suppose (G2) holds and suppose (3.1-3.3) has an eventually negative solution, then the following recurrence relation

$$
\Delta^{2}((n+1) W(n))+(n+1) p(n) \varphi(W(n)) \leq-G(n), \quad n \geq 0
$$

has an eventually positive solution.

Proof. From (3.1), we obtain

$$
\begin{aligned}
\Delta_{21}^{2} u(n-k, k) & =-c(n-k, k, u(n-k, k))+f(n-k, k) \\
& \leq-p(n) \varphi(u(n-k, k))+f(n-k, k) \\
& =-(n+1) p(n) \frac{\varphi(u(n-k, k))}{n+1}+f(n-k, k) .
\end{aligned}
$$

Substituting (3.7) into (3.5), we obtain

$$
\Delta^{2}((n+1) U(n)) \leq g(n+1)+h(n+1)-(n+1) p(n) \varphi(U(n))+\sum_{k=0}^{n} f(n-k, k)
$$

as desired. The rest of the proof is similar to that of Theorem 2.1.

In view of the above Theorem, in order to obtain oscillation theorems for (3.1), we need to consider recurrence relations of the following form

$$
\Delta^{2}((n+1) U(n))+(n+1) p(n) \varphi(U(n)) \leq \Psi(n), \quad n \geq 1
$$

A simple result is as follows, the proof of which is similar to that of Lemma 2.1. 

If

Lemma 3.1. Assume that $p(j) \geq 0$ for $j \geq 0$ and $\varphi$ is nonnegative on $(0, \infty)$.

$$
\liminf _{k \rightarrow \infty} \frac{1}{k-N+1} \sum_{n=N}^{k} \sum_{j=N}^{n} \Psi(j)=-\infty
$$

holds, then the recurrence relation (3.8) cannot have an eventually positive solution.

Lemma 3.2. Assume that $p(j) \geq 0$ for $j \geq 0$ and $\varphi$ is nonnegative and nondecreasing on $(0, \infty)$. Assume further that there is a sequence $\{v(n)\}$ which has a nonpositive subsequence $\left\{v\left(j_{k}\right)\right\}$ and satisfies $\Delta^{2} v(j)=\Psi(j)$ for all large $j$, and that

$$
\liminf _{k \rightarrow \infty} \frac{1}{k-N+1} \sum_{n=N}^{k} \sum_{j=N}^{n} \Psi(j)-(j+1) p(j) \varphi(\max (v(j), 0) /(j+1))=-\infty
$$

then (3.8) cannot have an eventually positive solution.

Proof. As in the proof of Lemma 2.2, if $\{U(n)\}$ is an eventually positive solution of (3.8), then the sequence $\{z(n)\}$ defined by $z(n)=(n+1) U(n)-v(n)$ is eventually positive and nondecreasing. Thus

$$
U(n)>\max \{v(n), 0\} /(n+1),
$$

and

$$
\varphi(U(n))>\varphi(\max \{v(n), 0\} /(n+1))
$$

for all large $n$. The rest of the proof is similar to that of Lemma 2.2, and is omitted.

Lemma 3.3. Assume that $p(j) \geq 0$ for $j \geq 0$ and $\varphi$ is nonnegative and nondecreasing on $(0, \infty)$. Assume further that there is a sequence $\{v(n)\}$ which has a nonpositive subsequence $\left\{v\left(n_{k}\right)\right\}$ and satisfies $\Delta^{2}((n+1) v(n))=\Psi(n)$ for all large $n$, and that

$$
\liminf _{n \rightarrow \infty}(n+1) v(n)=0 .
$$

If (3.8) has an eventually positive solution, so does the recurrence relation

$$
\Delta^{2}((n+1) W(n))+(n+1) p(n) \varphi(W(n)) \leq 0, \quad j \geq 0 .
$$

Proof. As in the proof of Lemma 2.3, if $\{U(j)\}$ is an eventually positive solution of (3.8), then the sequence $\{z(n)\}$ defined by $z(n)=(n+1) U(n)-(n+1) v(n)$ is eventually positive and increasing. After summing

$$
\Delta^{2}\{(n+1) U(n)-(n+1) v(n)\} \leq-(n+1) p(n) \varphi(U(n)),
$$


we obtain

$$
\Delta z(i) \geq \sum_{n=i}^{k}(n+1) p(n) \varphi(U(n))
$$

and thus

$$
\Delta z(i) \geq \sum_{n=i}^{\infty}(n+1) p(n) \varphi(U(n)) .
$$

Another summation then yields

$$
(k+2) U(k+1)-(k+2) v(k+1)=z(k+1) \geq z(K)+\sum_{i=K}^{k} \sum_{n=i}^{\infty}(n+1) p(n) \varphi(U(n)),
$$

where $K$ is taken to be a large integer so that $z(K)>0$. Since (3.11) holds, we have

$$
(k+2) U(k+1) \geq \frac{z(K)}{2}+\sum_{i=K}^{k} \sum_{n=i}^{\infty}(n+1) p(n) \varphi(U(n))
$$

for all large $k$. As in the proof of Lemma 2.3, the sequence $\{W(n)\}$ defined by

$$
(k+2) W(k+1)=\sum_{i=K}^{k} \sum_{n=i}^{\infty}(n+1) p(n) \varphi(U(n))
$$

is an eventually positive solution of (3.12).

The next lemma is similar to Lemma 2.4. Its proof will be omitted.

Lemma 3.4. Assume that $p(j) \geq 0$ for $j \geq 0$ and $\varphi$ is nonnegative and nondecreasing on $(0, \infty)$. Assume further that there is a sequence $\{v(j)\}$ which has a constant subsequence $\left\{v\left(j_{k}\right)\right\} \equiv\{h\}$ and $v(j) \geq h$ for all large $j$. Assume further that $\Delta^{2}((j+1) v(j))=\Psi(j)$ for all large $j$. If (3.8) has an eventually positive solution $\{U(j)\}$, then so does the recurrence relation (3.12).

To close this section, we remark that in view of the formal identity

$$
(n+1) \Delta^{2}(n x(n-1))=\Delta((n+1) n \Delta x(n-1)),
$$

the recurrence relation (3.8) is equivalent to

$$
\Delta((n+2)(n+1) \Delta U(n))+(n+2)(n+1) p(n) \varphi(U(n)) \leq(n+2) \Psi(n), \quad n \geq 1 .
$$

\section{Oscillation Criteria for Unforced Recurrence Relations I}

We have derived in the last two sections several sufficient conditions for no solutions of the forced recurrence relations (2.6) or (3.8) to be eventually positive. However, in 
Lemmas 2.3, 2.4, 3.3 and 3.4 , we see that additional oscillation criteria are also needed for the unforced recurrence relations

$$
\Delta^{2} x(j-1)+p(j) \varphi(x(j)) \leq 0, \quad j \geq 1
$$

and

$$
\Delta^{2}((j+1) y(j))+(j+1) p(j) \varphi(y(j)) \leq 0, \quad j \geq 0,
$$

or its equivalent form (in view of (3.13))

$$
\Delta((j+2)(j+1) \Delta y(j))+(j+2)(j+1) p(j) \varphi(y(j)) \leq 0, \quad j \geq 0,
$$

where $p$ is eventually nonnegative. Related problems have been considered by many authors in recent years (see for example $[4,5,9,10,14]$ ). Most of the results obtained by these authors are for nonlinear difference equations. Some of them can be modified to suit our recurrence relations. In this section, we shall derive several dissimilar oscillation criteria for (4.1). An oscillation criteria for (4.3), which is of independent interest, however, will be derived in the next section.

We first make the following observation.

Lemma 4.1. Suppose $p(j)$ is eventually nonnegative, and $\varphi$ is positive and nondecreasing on $(0, \infty)$. If $(4.1)$ has an eventually positive solution $\{x(j)\}$, so does the relation

$$
\left\llcorner^{2} z(j)+p(j) \varphi(z(j)) \leq 0, \quad j \geq 1\right.
$$

Proof. In view of (4.1), $\{x(j)\}$ is an eventually positive and concave sequence, and hence $\Delta x(j) \geq 0$ for all large $j$. Thus

$$
\Delta^{2} x(j)+p(j) \varphi(x(j)) \leq \Delta^{2} x(j)+p(j) \varphi(x(j+1)) \leq 0
$$

are required.

Theorem 4.1. Suppose $\varphi$ is a positive nondecreasing function on $(0, \infty)$ and $p(j)$ is eventually nonnegative such that

$$
\sum_{j=1}^{\infty} p(j)=\infty
$$

then (4.4) (and hence (4.1)) cannot have an eventually positive solution.

Proof. Suppose to the contrary that $\{x(n)\}$ is an eventually positive solution of (4.4). Then as we have seen before, $x(n)>0, \Delta x(n) \geq 0$ and $\Delta^{2} x(n) \leq 0$ for $n$ larger than or equal to some integer $N$. Let

$$
z(n)=\frac{\Delta x(n)}{\varphi(x(n))}, \quad n \geq N .
$$


Then

$$
\begin{aligned}
\Delta z(n) & =\frac{\Delta x(n+1)}{\varphi(x(n+1))}-\frac{\Delta x(n)}{\varphi(x(n))} \\
& =\frac{\Delta^{2} x(n)}{\varphi(x(n))}-\frac{\Delta x(n+1) \Delta \varphi(x(n))}{\varphi(x(n)) \varphi(x(n+1))} \\
& \leq \frac{\Delta^{2} x(n)}{\varphi(x(n))} \leq-p(n), \quad n \geq N,
\end{aligned}
$$

where we have used the fact that $\Delta x(n+1) \geq 0$ and $\Delta \varphi(x(n)) \geq 0$. Hence

$$
0<z(k+1) \leq z(N)-\sum_{n=N}^{k} p(n),
$$

which implies

$$
\sum_{n=N}^{\infty} p(n) \leq z(N) .
$$

This contradicts our assumption (4.5).

In case condition (4.5) does not hold, we can still derive several oscillation criteria when additional conditions hold.

Theorem 4.2. Suppose $\varphi$ is a positive nondecreasing function on $(0, \infty)$, and $p(j)$ is eventually nonnegative such that

$$
\begin{aligned}
& \sum_{j=1}^{\infty} p(j)<\infty \\
& \sum_{j=1}^{\infty} j p(j)=\infty
\end{aligned}
$$

and

$$
0<\int_{\varepsilon}^{\infty} \frac{d u}{\varphi(u)}<\infty \text { for some } \varepsilon>0
$$

then (4.4) (and hence (4.1)) cannot have an eventually positive solution.

Proof. Suppose to the contrary that $\{x(n)\}$ is an eventually positive solution of (4.4). As seen before, $x(n)>0, \Delta x(n) \geq 0$ and $\Delta^{2} x(n) \leq 0$ for $n$ larger than or equal to some integer $N$. Let

$$
z(n)=\frac{n \Delta x(n)}{\varphi(x(n))}, \quad n \geq N
$$

Then

$$
\begin{aligned}
\Delta z(n) & =\frac{(n+1) \Delta x(n+1)}{\varphi(x(n+1))}-\frac{n \Delta x(n)}{\varphi(x(n))} \\
& =\frac{n \Delta^{2} x(n)}{\varphi(x(n))}+\frac{\Delta x(n+1)}{\varphi(x(n+1))}-\frac{n \Delta x(n+1) \Delta \varphi(x(n))}{\varphi(x(n)) \varphi(x(n+1))} \\
& \leq \frac{n \Delta^{2} x(n)}{\varphi(x(n))}+\frac{\Delta x(n+1)}{\varphi(x(n+1))} \leq-n p(n)+\frac{\Delta x(n)}{\varphi(x(n+1))}
\end{aligned}
$$


where we have used the fact that $\Delta x(n+1) \geq 0$ and $\Delta \varphi(x(n)) \geq 0$ in derving the first inequality, and the fact that $\Delta x(n+1) \leq \Delta x(n)$ in the second. Hence by summing and then rearranging the resulting inequality, we obtain

$$
z(k+1)+\sum_{n=N}^{k} n p(n) \leq z(N)+\sum_{n=N}^{k} \frac{\Delta x(n)}{\varphi(x(n+1))} .
$$

In view of Lemma 1.2 and our assumptions, we arrive at

$$
\infty=\sum_{n=N}^{\infty} n p(n) \leq z(\infty)+\sum_{n=N}^{\infty} n p(n) \leq z(N)+\int_{x(N)}^{\infty} \frac{d u}{\varphi(u)}<\infty,
$$

which is a contradiction.

Theorem 4.3. Suppose $\varphi$ is a positive function on $(0, \infty)$ such that

$$
\varphi(x) / x \geq \delta>0 \quad \text { for } x \geq \alpha>0
$$
and suppose $p(j)$ is eventually nonnegative. If there is a positive sequence $\{\sigma(i)\}$
such that

$$
\sum_{i=N}^{\infty} p(i+1) \sigma(i)=\infty
$$

and

$$
\frac{\sum_{i=N-1}^{n} \frac{(\Delta \sigma(i-1))^{2}}{\sigma(i)}\left\{1+\frac{1}{i-N}\right\}}{\sum_{i=N}^{n} p(i+1) \sigma(i)} \rightarrow 0
$$

as $n \rightarrow \infty$, then (4.1) cannot have an eventually positive solution.

Proof. Suppose to the contrary that $\{x(n)\}$ is an eventually positive solution of (4.1). Then by our assumptions on $\varphi$, we see from (4.1) and Lemma 1.1 that $x(n)>0$, $\Delta x(n) \geq 0$ and $\Delta^{2} x(n) \leq 0$ for $n$ larger than or equal to some integer $N$. Let

$$
v(j)=\frac{\Delta x(j)}{x(j)}, \quad j \geq 1,
$$

which is nonnegative for $j \geq N$. Then from (4.1), we have

$$
\Delta v(j) \leq-\frac{p(j+1) \varphi(x(j+1))}{x(j+1)}-\frac{(\Delta x(j))^{2}}{x(j) x(j+1)} \leq-\delta p(j+1)-\frac{v^{2}(j)}{1+v(j)}
$$

which implies

$$
\delta p(j+1) \leq-\Delta v(j)-\frac{v^{2}(j)}{1+v(j)}
$$


Multiplying (4.15) by $\sigma(j)$ and summing the resulting equation, we obtain

$$
\begin{aligned}
& \delta \sum_{j=N}^{n} p(j+1) \sigma(j) \leq-\sum_{j=N}^{n} \sigma(j) \Delta v(j)-\sum_{j=N}^{n} \frac{\sigma(j) v^{2}(j)}{1+v(j)} \\
= & -\sigma(n) v(n+1)+\sigma(N-1) v(N)+\sum_{j=N}^{n} v(j) \Delta \sigma(j-1)-\sum_{j=N}^{n} \frac{\sigma(j) v^{2}(j)}{1+v(j)} \\
\leq & \sigma(N-1) v(N)-\sum_{j=N}^{n}\left\{\left[\frac{\sigma(j) v^{2}(j)}{1+v(j)}\right]^{1 / 2}-\frac{1}{2}\left[\frac{1+v(j)}{\sigma(j) v^{2}(j)}\right]^{1 / 2} v(j) \Delta \sigma(j-1)\right\}^{2} \\
& +\frac{1}{4} \sum_{j=N}^{n} \frac{(\Delta \sigma(j-1))^{2}}{\sigma(j)}(v(j)+1) \\
\leq & \sigma(N-1) v(N)+\frac{1}{4} \sum_{j=N}^{n} \frac{(\Delta \sigma(j-1))^{2}}{\sigma(j)}(v(j)+1) .
\end{aligned}
$$

Next, we note that $\Delta^{2} x(j) \leq 0$ for $j \geq N$ implies $\Delta x(j) \geq \Delta x(j+1)$ for $j \geq N$. Thus

$$
\frac{(\Delta x(j))^{2}}{x(j) x(j+1)} \geq \frac{\Delta x(j) \Delta x(j+1)}{x(j) x(j+1)}
$$

which implies, in view of (4.14), that

$$
\Delta v(j)+v(j) v(j+1) \leq 0, \quad j \geq N
$$

Therefore,

$$
\Delta\left\{j-\frac{1}{v(j)}\right\} \leq 0, \quad j \geq N
$$

and

$$
v(n) \leq \frac{1}{n-N}, \quad n \geq N
$$

Substituting (4.17) into (4.16), we obtain

$$
\delta \sum_{j=N}^{n} p(j+1) \sigma(j) \leq \sigma(N-1) v(N)+\frac{1}{4} \sum_{j=N}^{n} \frac{(\Delta \sigma(j-1))^{2}}{\sigma(j)}\left\{1+\frac{1}{j-N}\right\} .
$$

Dividing through the above inequality by the left hand side and then taking limits as $n \rightarrow \infty$, we obtain, in view of the assumptions of our Theorem, the inequality $1 \leq 0$, which is a contradiction.

Suppose we let $\sigma(i)=i^{\tau}$ for $i \geq 1$, where $0<\tau<1$. Then it is easy to see that

$$
\frac{(\Delta \sigma(i-1))^{2}}{\sigma(i)} \leq \frac{\tau^{2}}{i^{\tau}(i-1)^{2-2 \tau}}
$$


which implies

$$
\sum_{i=N}^{\infty} \frac{(\Delta \sigma(i-1))^{2}}{\sigma(i)}\left\{1+\frac{1}{i-N}\right\}<\infty
$$

The following is now clear.

Corollary 4.1. Suppose $\varphi$ is a positive function on $(0, \infty)$ such that $(4.11)$ holds and suppose $p(j)$ is eventually nonnegative. Suppose further that for some $\tau \in(0,1)$,

$$
\sum_{i=N}^{\infty} i^{\tau} p(i+1)=\infty
$$

Then (4.1) cannot have an eventually positive solution.

Under the assumptions of Theorem 4.3, we have seen in the proof that the sequence $\{v(j)\}$ is eventually nonnegative and that (4.14) holds. Summing (4.14) from $s$ to $n$, we obtain

$$
v_{n+1}+\delta \sum_{j=s}^{n} p(j+1)+\sum_{j=s}^{n} \frac{v^{2}(j)}{1+v(j)} \leq v(s), \quad n \geq s \geq N
$$

which implies

$$
\delta \sum_{j=s}^{n} p(j+1) \leq v(s)<\infty, \quad s \geq N
$$

If we now write

$$
Q_{k}^{(0)}=\delta \sum_{j=k}^{n} p(j+1), \quad k=0,1,2, \ldots
$$

and

$$
Q_{k}^{(1)}=Q_{k}^{(0)}+\sum_{i=k}^{\infty} \frac{\left(Q_{i}^{(0)}\right)^{2}}{1+Q_{i}^{(0)}}, \quad k=0,1,2, \ldots,
$$

then, in view of (4.19) and (4.20), we have

$$
Q_{s}^{(1)} \leq Q_{s}^{(0)}+\sum_{i=s}^{n} \frac{v^{2}(i)}{1+v(i)} \leq v(s)<\infty, \quad s \geq N .
$$

Similarly, if we define for $j \geq 1$,

$$
Q_{k}^{(j+1)}=Q_{k}^{(0)}+\sum_{i=k}^{\infty} \frac{\left(Q_{i}^{(j)}\right)^{2}}{1+Q_{i}^{(j)}}, \quad k=0,1,2, \ldots
$$

Then assume by induction that $Q_{k}^{(j)} \leq v(k)$ for $k \geq N$, we have

$$
Q_{s}^{(j+1)} \leq Q_{s}^{(0)}+\sum_{i=s}^{n} \frac{v^{2}(i)}{1+v(i)} \leq v(s)<\infty, \quad s \geq N .
$$


The following Theorem is now clear.

Theorem 4.4. Suppose $\varphi$ is a positive function on $(0, \infty)$, and suppose $p(j)$ is eventually nonnegative. If (4.1) has an eventually positive solution, then the double sequence $\left\{Q_{k}^{(j)}\right\}$ defined by $(4.20-4.22)$ satisfies $Q_{k}^{(j)}<\infty$ for every $j \geq 0$ and $k$ larger than or equal to some integer $N$.

Corollary 4.2. Under the same assumptions of Theorem 4.4, if

$$
\sum_{j=N}^{\infty} p(j+1)=\infty
$$

or

$$
\delta \sum_{j=n}^{\infty} p(j+1) \geq \frac{1}{n}
$$

then (4.1) cannot have an eventually positive solution.

Proof. We have already seen that if (4.1) has an eventually positive solution, then (4.19) holds. Next, note that if (4.23) holds, then

$$
Q_{k}^{(1)} \geq \frac{1}{k}+\sum_{i=k}^{\infty} \frac{\left(Q_{i}^{(0)}\right)^{2}}{1+Q_{i}^{(0)}} \geq \frac{1}{k}+\sum_{i=k}^{\infty} \frac{1}{i(i+1)}=\frac{2}{k} .
$$

By induction, it is not difficult to see that

$$
Q_{k}^{(j)} \geq \frac{j+1}{k}, \quad j \geq 0
$$

Thus $Q_{M}^{(j)} \rightarrow \infty$ as $j \rightarrow \infty$ for any fixed large integer $M$. This shows that (4.1) cannot have an eventually positive solution.

Our final Theorem in this section applies to functions of the form $\varphi(x)=x^{\tau}$ where $\tau$ is any number between 0 and 1 .

Theorem 4.5. Let $\varphi$ be a positive nondecreasing function defined on $(0, \infty)$ such that

$$
\varphi(\alpha x) \geq \varphi(\alpha) \varphi(x) \text { for } x>0 \text { and all large } \alpha,
$$

and that

$$
\int_{+0}^{\delta} \frac{d s}{\varphi(s)}<\infty \quad \text { for some } \delta>0 .
$$

Suppose further that $p(j)$ is eventually positive and

$$
\sum_{j=N}^{\infty} p(j) \varphi(j / 2)=\infty
$$


Then the recurrence relation (4.1) cannot have an eventually positive solution.

Proof. Suppose to the contrary that $\{x(n)\}$ is an eventually positive solution of (4.1). As we have seen before, we may infer from Lemma 1.1 and (4.1) that $x(n)>0$, $\Delta x(n)>0$ and $\Delta^{2} x(n)<0$ for $n$ larger than or equal to some integer $N \geq 2$. It is also easy to see that (see for example [5, Lemma 4.1])

$$
x(n) \geq \frac{n}{2} \Delta x(n-1) \geq \frac{n}{2} \Delta x(n), \quad n \geq 2 N .
$$

Thus, in view of (4.1) and (4.24),

$$
\frac{\Delta^{2} x(j-1)}{\varphi(\Delta x(j-1))}+p(j) \varphi(j / 2) \leq \frac{\Delta^{2} x(j-1)}{\varphi(\Delta x(j))}+p(j) \frac{\varphi(x(j))}{\varphi(\Delta x(j))} \leq 0, \quad j \geq 2 N .
$$

Summing the above inequality from $2 N$ to $k$, we obtain

$$
\sum_{j=2 N}^{k} \frac{\Delta^{2} x(j-1)}{\varphi(\Delta x(j-1))}+\sum_{j=2 N}^{k} p(j) \varphi(j / 2) \leq 0 .
$$

In view of Lemma 1.2 and (4.25), we have

$$
-\infty<\int_{\Delta x(2 N-1)}^{\Delta x(k)} \frac{d s}{\varphi(s)} \leq \sum_{j=2 N}^{k} \frac{\Delta^{2} x(j-1)}{\varphi(\Delta x(j-1))} \leq-\sum_{j=2 N}^{k} p(j) \varphi(j / 2), \quad k \geq 2 N,
$$

which contradicts the condition (4.26).

\section{Oscillation Criteria for Unforced Recurrence Relation II}

In this section, we shall derive an oscillation criterion for the recurrence relation

$$
\Delta(r(n) \Delta u(n))+a(n) f(u(n)) \leq 0, \quad n \geq 0,
$$

where $r(n)>0, a(n) \geq 0$ for $n \geq 0$ and $f$ is a positive nondecreasing function defined on $(0, \infty)$. As a particular application, this result reduces to an oscillation criterion for the recurrence relation (4.3) as promised in the last section.

Theorem 5.1. (cf. [8, Theorem 2]) Suppose there is a positive sequence $\{\sigma(n)\}$ which satisfies $\Delta \sigma(n) \leq 0$ and $\Delta(r(n) \Delta \sigma(n)) \geq 0$ for $n \geq 0$ and

$$
\begin{gathered}
\sum^{\infty} \frac{1}{\sigma(n+1) r(n)}=\infty, \\
\sum^{\infty} \sigma(n) a(n)=\infty .
\end{gathered}
$$


Suppose further that

$$
\int_{+0}^{\delta} \frac{d t}{f(t)}<\infty \quad \text { for some } \delta>0 .
$$

Then (5.1) cannot have an eventually positive solution.

Proof. Let $\{u(n)\}$ be an eventually positive solution of (5.1), then

$$
\Delta(r(n) \Delta u(n)) \leq-a(n) f(u(n)) \leq 0,
$$

for all large $n$. Thus $r(n) \Delta u(n)$ is eventually of constant sign. Multiply (5.1) by $\sigma(n) / f(u(n))$ and sum the resulting inequality form $N$ to $k$, we obtain

$$
\begin{gathered}
\frac{\sigma(k) r(k+1) \Delta u(k+1)}{f(u(k))}+\sum_{n=N}^{k} \frac{r(n) \sigma(n) \Delta u(n) \Delta f(u(n))}{f(u(n)) f(u(n+1))} \\
\leq \frac{\sigma(N-1) \sigma(N) \Delta u(N)}{f(u(N-1))}+\sum_{n=N}^{k} \frac{r(n) \Delta u(n) \Delta \sigma(n)}{f(u(n+1))}-\sum_{n=N}^{k} a(n) \sigma(n),
\end{gathered}
$$

for some large integer $N$. Note that since $\Delta u(n) \Delta f(u(n)) \geq 0$ for all large $n$, the sum on the left hand side of the above inequality is nonnegative. We now have two cases to consider. Suppose first that $\Delta u(n) \geq 0$ for all large $n$. Then the left hand side is nonnegative and the first sum on the right hand side is nonpositive. Thus by letting $k \rightarrow \infty$, we get a contradiction in view of (5.3).

Next, suppose $\Delta u(n) \leq 0$ for all large $n$. We assert that the first sum on the right hand side of the above inequality is bounded above. Indeed,

$$
\begin{aligned}
\sum_{n=N}^{k} \frac{r(n) \Delta u(n) \Delta \sigma(n)}{f(u(n+1))} & \leq r(N) \Delta \sigma(N) \sum_{n=N}^{k} \frac{\Delta u(n)}{f(u(n+1))} \\
& \leq r(N) \Delta \sigma(N) \int_{u(N)}^{u(k+1)} \frac{d s}{f(s)}
\end{aligned}
$$

where we have used Lemma 1.2 in deriving the second inequality. Since the integral in (5.6) is bounded in view of (5.4), our assertion is proved.

Since the second sum on the right hand side of (5.5) is unbounded, we may assume $k$ is so large that the right hand side of (5.5) is less than -1 , so that

$$
\frac{\sigma(k) r(k+1) \Delta u(k+1)}{f(u(k))}+\sum_{n=N}^{k} \frac{r(n) \sigma(n) \Delta u(n) \Delta f(u(n))}{f(u(n)) f(u(n+1))} \leq-1 .
$$

Since $\Delta \sigma(n) \leq 0$ and $f$ is nondecreasing, we obtain

$$
\frac{\sigma(k+2) r(k+1) \Delta u(k+1)}{f(u(k+1))}+\sum_{n=N}^{k} \frac{r(n) \sigma(n) \Delta u(n) \Delta f(u(n))}{f(u(n)) f(u(n+1))} \leq-1
$$


for all large $k$. Thus

$$
\begin{aligned}
& \sum_{k=T}^{i} \frac{1}{\sigma(k+2) r(k+1)} \sum_{n=N}^{k} \frac{r(n) \sigma(n) \Delta u(n) \Delta f(u(n))}{f(u(n)) f(u(n+1))}+\sum_{k=T}^{i} \frac{\Delta u(k+1)}{f(u(k+1))} \\
\leq & \sum_{k=T}^{i} \frac{-1}{\sigma(k+2) r(k+1)} .
\end{aligned}
$$

Since the double sum in (5.7) is nonnegative, thus in view of Lemma 1.2, we have

$$
\int_{u(T+1)}^{u(i+2)} \frac{d t}{f(t)} \leq \sum_{k=T}^{i} \frac{\Delta u(k+1)}{f(u(k+1))} \leq \sum_{k=T}^{i} \frac{-1}{\sigma(k+2) r(k+1)} .
$$

The integral in (5.8) is bounded below by means of (5.4), thus letting $i \rightarrow \infty$, we obtain a contradiction in view of (5.2).

As an immediate corollary, suppose

$$
\sum_{i=0}^{\infty} \frac{1}{r(i)}<\infty
$$

Then letting

$$
\sigma(n)=\sum_{i=n}^{\infty} \frac{1}{r(i)}, \quad n \geq 0
$$

we have $\sigma(n)>0, \Delta \sigma(n)=-1 / r(n)$ and $\Delta(r(n) \Delta \sigma(n))=\Delta(-1)=0$ for $n \geq 0$. Furthermore, (5.2) holds in view of Lemma 1.3. The following is now easily seen.

Corollary 5.1. Suppose (5.3), (5.4) and (5.9) hold, then (5.1) cannot have an eventually positive solution.

We now turn our attention to the recurrence relation

$$
\Delta((n+2)(n+1) y(n))+(n+2)(n+1) p(n) \varphi(y(n)) \leq 0, \quad n \geq 0 .
$$

Note that

$$
\sum^{\infty} \frac{1}{(n+1) n}<\infty
$$

Thus by means of Corollary 5.1, we have the following result.

Theorem 5.2. Suppose $p(n) \geq 0$ for $n \geq 0$ and $\varphi$ is a positive nondecreasing function defined on $(0, \infty)$. Suppose further that

$$
\sum_{n=0}^{\infty}(n+2) p(n)=\infty \quad \text { and } \quad \int_{+0}^{\delta} \frac{d t}{\varphi(t)}<\infty \text { for some } \delta>0
$$


then the recurrence relation (5.10) cannot have an eventually positive solution.

\section{Oscillation Criteria for Initial Boundary Value Problems}

According to the results in the previous Sections, it is now easy to obtain oscillation criteria for the initial boundary problem (2.1-2.3). We illustrate the principle for obtaining such oscillation criteria as follows. If the following conditions hold:

(i) $p(j) \geq 0$ for $j \geq 0$;

(ii) $\varphi$ is a nonnegative on $(0, \infty)$; and

(iii) $\liminf \operatorname{in}_{k \rightarrow \infty} \frac{1}{k-N+1} \sum_{n=N}^{k} \sum_{j=N}^{n} F(j)=-\infty$,

where $F(j)$ is defined in Theorem 2.1. Then by means of Lemma 2.1, (2.5) cannot have an eventually positive solution. If in addition the condition $(\mathrm{H} 1)$ holds, then in view of Theorem 2.1, (2.1-2.3) cannot have an eventually positive solution.

Similarly, if (i), (ii) and

$$
\limsup _{k \rightarrow \infty} \frac{1}{k-N+1} \sum_{n=N}^{k} \sum_{j=N}^{n} F(j)=\infty,
$$

then

$$
\Delta^{2} W(j-1)+p(j) \varphi(U(j)) \leq-F(j), \quad j \geq 0
$$

cannot have an eventually positive solution. Furthermore, suppose the additional conditions ( $\mathrm{H} 1$ ) and (H2) hold, then (2.1-2.3) cannot have an eventually negative solution. The following is now clear.

Theorem 6.1. Every solution of $(2.1-2.3)$ is oscillatory provided $(\mathrm{H} 1-\mathrm{H} 2)$ together with the following conditions hold:

$$
\liminf _{k \rightarrow \infty} \frac{1}{k-N+1} \sum_{n=N}^{k} \sum_{j=N}^{n} F(j)=-\infty
$$

and

$$
\limsup _{k \rightarrow \infty} \frac{1}{k-N+1} \sum_{n=N}^{k} \sum_{j=N}^{n} F(j)=\infty .
$$

As another example, we can derive the following oscillation criteria from Lemma 2.2 and Theorem 2.1.

Theorem 6.2. Every solution of $(2.1-2.3)$ is oscillatory provided $(\mathrm{H} 1-\mathrm{H} 3)$ together with the following conditions hold: $j \geq 0$

(i) there is an oscillatory sequence $\{v(j)\}$ such that $\Delta^{2} v(j-1)=F(j)$ for 
(ii) $\liminf _{k \rightarrow \infty} \frac{1}{k-N+1} \sum_{n=N}^{k} \sum_{j=N}^{n} F(j)-p(j) \varphi(\max (v(j), 0))=-\infty$; and

(iii) $\limsup _{k \rightarrow \infty} \frac{1}{k-N+1} \sum_{n=N}^{k} \sum_{j=N}^{n} F(j)+p(j) \varphi(\max (-v(j), 0))=\infty$.

The following theorem is derived from Lemma 2.3 and Theorem 2.1.

Theorem 6.3. Every solution of $(2.1-2.3)$ is oscillatory provided $(\mathrm{H} 1-\mathrm{H} 3)$ together with the following conditions hold: $j \geq 0$;

(i) there is an oscillatory sequence $\{v(j)\}$ such that $\Delta^{2} v(j-1)=F(j)$ for

(ii) $\lim _{j \rightarrow \infty} v(j)=0$; and

(iii) the recurrence relation

$$
\Delta^{2} x(j-1)+p(j) \varphi(x(j)) \leq 0, \quad j \geq 0
$$

does not have an eventually positive solution.

We remark that condition (iii) in the above Theorem can be replaced by oscillation criteria for the recurrence relation (6.1). For instance, in view of Theorem 4.1, we have the following corollary.

Corollary 6.1. Every solution of $(2.1-2.3)$ is oscillatory provided $(\mathrm{H} 1-\mathrm{H} 4)$, (4.5) together with the following conditions hold:

(i) there is an oscillatory sequence $\{v(j)\}$ such that $\Delta^{2} v(j-1)=F(j)$ for $j \geq 0 ;$ and

(ii) $\lim _{j \rightarrow \infty} v(j)=0$.

Corollary 6.2. Every solution of $(2.1-2.3)$ is oscillatory provided $(\mathrm{H} 1-\mathrm{H} 4)$, (4.8 - 4.10) together with the following conditions hold:

(i) there is an oscillatory sequence $\{v(j)\}$ such that $\Delta^{2} v(j-1)=F(j)$ for $j \geq 0$; and

(ii) $\lim _{j \rightarrow \infty} v(j)=0$.

The final result in this section is derived from Lemma 2.4 and Theorem 2.1.

Theorem 6.4. Every solution of $(2.1-2.3)$ is oscillatory provided $(\mathrm{H} 1-\mathrm{H} 3)$ together with the following conditions hold:

(i) there is a sequence $\{v(j)\}$ such that $\Delta^{2} v(j-1)=F(j)$ for $j \geq 0$;

(ii) $\{v(j)\}$ has two constant subsequence $\left\{h_{1}\right\}$ and $\left\{h_{2}\right\}$ and $h_{1} \leq v(j) \leq h_{2}$ for $j \geq 0 ;$ and

(iii) the recurrence relation

$$
\Delta^{2} x(j-1)+p(j) \varphi(x(j)) \leq 0, \quad j \geq 0
$$

does not have an eventually positive solution: 


\section{Oscillation Criteria for Characteristic Initial Value Problems}

As in the last Section, it is now relatively easy to derive oscillation criteria for the problem (3.1-3.3). In what follows, the function $G(j)$ is the one defined in Theorem 3.1.

Theorem 7.1. Every solution of $(3.1-3.3)$ is oscillatory provided (G1-G2) together with the following conditions hold:

(i) $\liminf \operatorname{in}_{k \rightarrow \infty} \frac{1}{k-N+1} \sum_{n=N}^{k} \sum_{j=N}^{n} G(j)=-\infty$; and

(ii) $\lim \sup _{k \rightarrow \infty} \frac{1}{k-N+1} \sum_{n=N}^{k} \sum_{j=N}^{n} G(j)=\infty$.

Theorem 7.2. Every solution of $(3.1-3.3)$ is oscillatory provided (G1-G3) together with the following conditions hold:

(i) there is an oscillatory sequence $\{v(j)\}$ which satisfies $\Delta^{2} v(j)=G(j)$ for $j \geq 1$; and

(ii) $\liminf \operatorname{in}_{k \rightarrow \infty} \frac{1}{k-N+1} \sum_{n=N}^{k} \sum_{j=N}^{n} G(j)-(j+1) p(j) \varphi(\max (v(j), 0) /(j+1))=-\infty$;

(iii) $\lim \sup _{k \rightarrow \infty} \frac{1}{k-N+1} \sum_{n=N}^{k} \sum_{j=N}^{n} G(j)+(j+1) p(j) \varphi(\max (-v(j), 0) /(j+1))=$ $\infty$.

Theorem 7.3. Every solution of $(3.1-3.3)$ is oscillatory provided (G1-G3) together with the following conditions hold:

(i) there is an oscillatory sequence $\{v(j)\}$ such that $\Delta^{2}((n+1) v(n))=G(n)$ for $n \geq 1$;

(ii) $\lim _{n \rightarrow \infty}(n+1) v(n)=0$; and

(iii) the recurrence relation

$$
\Delta((n+2)(n+1) \Delta x(n))+(n+2)(n+1) \varphi(u(n)) \leq 0, \quad n \geq 1
$$

does not have an eventually positive solution.

Theorem 7.4. Every solution of $(3.1$ - 3.3) is oscillatory provided (G1-G3) together with the following conditions hold:

(i) there is a sequence $\{v(n)\}$ which satisfies $\Delta^{2}((n+1) v(n))=G(n)$ for $n \geq 1$;

(ii) $\{v(n)\}$ has two constant subsequence $\left\{h_{1}\right\}$ and $\left\{h_{2}\right\}$ such that $h_{1} \leq v(j) \leq$ $h_{2}$ for all large $j$; and

(iii) the recurrence relation

$$
\Delta((n+2)(n+1) \Delta x(n))+(n+2)(n+1) \varphi(u(n)) \leq 0, \quad n \geq 1
$$

does not have an eventually positive solution.

The last result follows Theorem 7.6 and Theorem 5.2.

Theorem 7.5. Every solution of $(3.1-3.3)$ is oscillatory provided (G1-G4) together with the following conditions hold: 
(i) there is a sequence $\{v(n)\}$ which satisfies $\Delta^{2}((n+1) v(n))=G(n)$ for $n \geq 1$;

(ii) $\{v(n)\}$ has two constant subsequence $\left\{h_{1}\right\}$ and $\left\{h_{2}\right\}$ such that $h_{1} \leq v(j) \leq$ $h_{2}$ for all large $j$;

(iii) $\sum^{\infty}(n+2) p(n)=\infty$; and

(iv) $\int_{+0}^{\delta} \frac{d t}{\varphi(t)}<\infty$ for some $\delta>0$.

\section{Acknowledgment}

The first author is supported by the NSC of R.O.C. and the second author is supported by the NNSF of China.

\section{References}

[1] S. S. Cheng and B. G. Zhang, "Qualitative theory of partial difference equations (I): Oscillation of nonlinear partial difference equations," Tamkang J. Math., 25, 279-288, 1994.

[2] S. S. Cheng, S. L. Xie and B. G. Zhang, "Qualitative theory of partial difference equations (II): Oscillation criteria for direct control systems in several variables," Tamkang J. Math., 26, 65-79, 1995.

[3] S. S. Cheng, B. G. Zhang and S. L. Xie, "Qualitative theory of partial difference equations (III): Forced oscillations of parabolic type partial difference equations," Tamkang J. Math., 26, 177-192, 1995.

[4] S. S. Cheng, T. C. Yan and H. J. Li, "Oscillation criteria for second order difference equations," Funkcialaj Ekvacioj, 34, 223-239, 1991.

[5] J. W. Hooker and W. T. Patula, "A second order nonlinear difference equations: oscillation and asymptotic behavior, J. Math. Anal. Appl., 91, 9-29, 1983.

[6] K. Kreith, T. Kusano and N. Yoshida, "Oscillation properties of nonlinear hyperbolic equations," SIAM J. Math. Anal., 15, 570-578, 1984.

[7] K. Kreith and G. Pagan, "Qualitative theory for hyperbolic characteristic initial value problems," Proc. Roy. Soc. Edinburg Sect.A, 94, 15-24, 1983.

[8] T. Kusano and H. Onose, "Oscillation theorems for second order differential equations with retarded argument," Proc. Japan Acad., 50, 342-346, 1974.

[9] H. J. Li and S. S. Cheng, "Asymptotically monotone solutions of a nonlinear difference equation," Tamkang J. Math., 24, 269-282, 1993.

[10] B. Szmanda, "Oscillation criteria for second order nonlinear difference equations," Annales Polonici Math., 43, 225-235, 1983.

[11] N. Yoshida, "On the zeros of solutions to nonlinear hyperbolic equations," Proc. Roy. Soc. Edinburg, 106A; 121-129, 1987.

[12] N. Yoshida, "An oscillation theorem for characteristic initial value problems for nonlinear hyperbolic equations," Proc. Amer. Math. Soc., 76, 95-100, 1979.

[13] B. G. Zhang and S. S. Cheng, "Oscillation criteria for delay difference equations," Fasciculi Math., 25, 13-32, 1995.

[14] B. G. Zhang, "Oscillation and asymptotic behavior of second order difference equations," J. Math. Anal. Appl., 173, 58-68, 1993.

Department of Mathematics, Tsing Hua University, Hsinchu, Taiwan, 30043.

Department of Mathematics, Ocean University of Qingdao, Qingdao, China 266003.

Department of Automation, South China University of Technology, Guangzhou, China, 510641. 